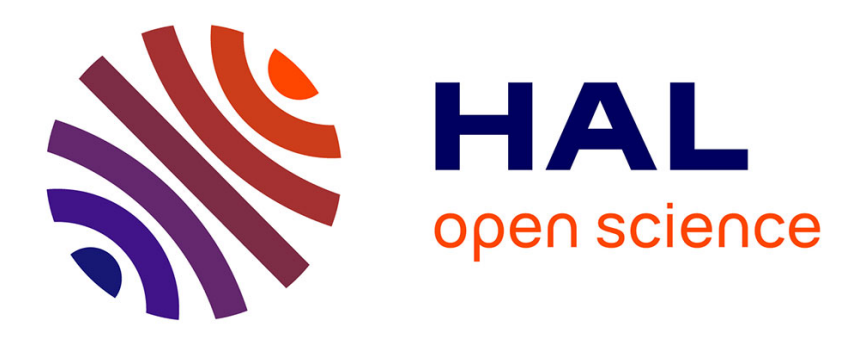

\title{
La protection de la plage par le droit de l'urbanisme
} Jean-Pierre Ferrand

\section{To cite this version:}

Jean-Pierre Ferrand. La protection de la plage par le droit de l'urbanisme. "Sous la plage, le droit ", Apr 2013, Toulon, France. hal-01080019

\section{HAL Id: hal-01080019 https://hal.science/hal-01080019}

Submitted on 4 Nov 2014

HAL is a multi-disciplinary open access archive for the deposit and dissemination of scientific research documents, whether they are published or not. The documents may come from teaching and research institutions in France or abroad, or from public or private research centers.
L'archive ouverte pluridisciplinaire HAL, est destinée au dépôt et à la diffusion de documents scientifiques de niveau recherche, publiés ou non, émanant des établissements d'enseignement et de recherche français ou étrangers, des laboratoires publics ou privés. 


\title{
LA PROTECTION DE LA PLAge PAR LE DROIT DE L'URBANISME
}

Par Jean-Pierre FERRAND

MCF/HDR

Aix-Marseille Université

\section{Plan}

I. La protection renforcée de la plage par les règles d'inconstructibilité

A. La protection de la plage en tant qu'espace, site, paysage remarquable ou caractéristique ........................... 6

B. La protection de la plage située dans la bande littorale des 100 mètres................................................

C. La protection de la plage par des restrictions à la réalisation de routes sur le littoral ................................. 11

II. La protection en devenir de la plage par l'urbanisme prévisionnel .......................................................... 12

A. La plage dans les documents d'urbanisme spécifiques .................................................................. 12

B. La plage dans les documents d'urbanisme généraux .................................................................. 14

\begin{abstract}
"Comme un animal vieillissant dont la carapace s'épaissit, formant autour de son corps une croûte imperméable qui ne permet plus à l'épiderme de respirer et accélère ainsi le progrès de sa sénescence, la plupart des pays européens laissent leurs côtes s'obstruer de villas, d'hôtels et de casinos. Au lieu que le littoral ébauche, comme autrefois, une image anticipée des solitudes océaniques, il devient une sorte de front où les hommes mobilisent périodiquement toutes leurs forces, pour donner l'assaut à une liberté dont ils démentent l'attrait par les conditions dans lesquelles ils acceptent de se la ravir. Les plages, où la mer nous livrait les fruits d'une agitation millénaire, étonnante galerie où la nature se classait toujours à l'avant-garde, sous le piétinement des foules ne servent plus guère qu'à la disposition et à l'exposition des rebuts. "
\end{abstract}

Claude LEVI-STRAUSS, Tristes Tropiques, Poche Terre Humaine, 1955.

«La plage est un espace convoité » expliquaient en 2009 le Conseil général de l'environnement et du développement durable (CGEDD) et l'inspection générale de l'administration (IGA) dans un rapport conjoint sur les difficultés d'application du décret relatif aux concessions de plage $\left({ }^{1}\right)$. Difficile aujourd'hui de ne pas réitérer une constatation de même nature tant les pressions purement urbanistiques comme économiques restent fortes sur ces zones particulières $\left({ }^{2}\right)$. Même si ces tensions n'ont jamais atteint la même intensité sur l'ensemble des zones littorales, l'attractivité de ces espaces fragiles est restée très forte $\left({ }^{3}\right)$, ainsi qu'en attestent les vives oppositions apparues à propos de certaines exploitations à caractère marchand ou le développement de phénomènes de cabanisation. La plage reste un lieu de tension où s'expriment des intérêts fortement contradictoires. La plage est ainsi non seulement un espace convoité mais aussi un espace très largement en danger. Même si la science est encore aujourd'hui dans l'incapacité d'en prévoir précisément les effets à moyen ou long terme, le risque de submersion maritime ne relève plus désormais de la simple supputation. Ce risque a désormais atteint un niveau de certitude qui impose de le prendre en compte notamment dans les documents d'urbanisme $\left({ }^{4}\right)$.

\footnotetext{
${ }^{1}$ CGEDD et IGA, Les difficultés d'application du décret relatif aux concessions de plage, Rapport de Mission, La documentation Française, 2009, p. 1.

${ }^{2}$ Il suffit pour s'en convaincre d'un bref aperçu de la presse locale. Le terme "paillotes » a ainsi acquis une renommée qui dépasse largement le cadre traditionnel des régions méditerranéennes.

${ }^{3}$ Voir sur la dimension sociologique de la plage en tant qu'objet " chaud ", V. Coëffé, " La plage, fabrique d'une touristi(cité) idéale ", L'information géographique, 2010/3 Vol.74, p.51-68.

${ }^{4}$ Voir sur cette question, Vanessa Mulot, Anne-Laure Vigneron et Marie-Laure Lambert-Hadid, " Le littoral face aux changements climatiques ", Méditerranée [En ligne], 115 | 2010, mis en ligne le 30 décembre 2012, consulté le 29 septembre 2013. URL: http://mediterranee.revues.org/5264.
} 
Pour autant la réponse du droit de l'urbanisme apparaît à la fois imprécise, multiple et souvent illisible. même si, de manière quelque peu paradoxale, la protection qu'il assure est à bien des égards souvent efficace.

La première difficultée rencontrée tient au fait que le droit de l'urbanisme ne définit pas précisément ce qu'est une plage $\left({ }^{5}\right)$. Comme l'indiquait en $2006 \mathrm{Ch}$. Maugüe "la notion de plage ne se trouve définie par aucun texte. Aucune disposition du code de l'urbanisme, y compris celles issues de la loi littoral, ne donne de définition des plages " $\left.{ }^{6}\right)$. On peut même ajouter que seules huit dispositions du code de l'urbanisme font référence explicitement au concept de plage $\left({ }^{7}\right)$, sans que d'ailleurs soit explicité le sens à donner à ce terme $\left({ }^{8}\right)$. C'est sans doute bien peu pour un « objet du droit » aussi présent dans le langage commun.

Dès lors, c'est plus par approximation que l'on peut essayer de définir ce terme $\left({ }^{9}\right)$. Ainsi, dans le langage courant, si le Littré voit dans la plage un "espace plat d'une étendue plus ou moins grande sur le rivage de la mer, et qui n'est recouvert d'eau que dans les grandes marées ", l'Académie française retient une définition plus large, "grève, rivage bordant la mer, couvert de sable ou de galets ", et sans doute plus conforme à ce qu'envisage le droit de l'urbanisme. Dans les deux définitions cependant, apparaît la référence au rivage dont le lien avec la plage apparaît essentiel sans toutefois que les deux notions soient identiques, se recoupent voire s'englobent.

Si le juge administratif n'a guère apporté à la définition juridique de la plage, l'administration, elle, a retenu une définition largement contestable ou pour le moins perfectible. En effet, le Ministère chargé de l'Écologie, développement et aménagement durables, saisi de cette question dans le cadre de l'application du décret du 26 mai 2006 relatif aux concessions de plage, a ainsi précisé que "Les plages, qu'elles soient naturelles ou artificielles, font partie intégrante du domaine public maritime naturel défini par l'article L. 2111-4 du code général de la propriété des personnes publiques. Ainsi, les plages entrent dans le cadre du "rivage " et des "lais et relais de mer ". Toutefois, ces éléments de définition de l'article L. 2111-4 sont plus larges que la notion de plage. Ces dernières sont plus particulièrement des parcelles en bord de mer qui sont recouvertes, par intermittence, totalement ou partiellement par les flots. Leur sol est généralement recouvert de sable, de graviers ou de galets. Ainsi, les plages relèvent plus d'une définition géologique que juridique ». $\left({ }^{10}\right)$

Si l'insuffisance de la définition purement juridique de la plage et l'utilité de la géologie (plus précisément sans doute de la géomorphologie) ne sont guère contestables, l'affirmation selon laquelle les plages font nécessairement partie intégrante du domaine public maritime n'emporte pas l'adhésion. En premier lieu, l'appartenance des plages au seul domaine public maritime est inexacte. Comme l'indiquait le Conseil d'Etat dans la jurisprudence Gozzoli, une partie de la plage peut parfaitement relever de la propriété d'une autre collectivité publique et à ce titre ne pas appartenir au domaine public maritime $\left({ }^{11}\right)$. On a tendance en effet à limiter exagérément la plage à l'estran $\left({ }^{12}\right)$, alors qu'elle

\footnotetext{
${ }^{5}$ Un certain nombre d'auteurs ont d'ailleurs fait un constat identique pour le littoral. Voir en ce sens, L. Prieur, « L'écriture des plans locaux d'urbanisme littoraux, Fiche introductive ", GRIDAUH, http://www.gridauh.fr/comptes-rendus-de-travaux/ecrituredes-plu/, consulté le 10 septembre 2013 ou B. Bousquet, " Définition et identification du littoral contemporain », RJE 1990, p.451. ${ }^{6}$ C. Maugüe, "La réaffirmation du caractère exceptionnel de l'occupation privative des plages », AJDA, 2006, p. 1496.

${ }^{7}$ Articles L.146-6 et R.146-1, articles L.146-6-1 et R.146-3 relatifs aux schémas d'aménagement des plages, article L.146-7 pour la création de routes sur les plages, articles L.156-2, L.156-3 et L.156-4 concernant certaines collectivités ultra marines.

${ }^{8}$ On pourrait d'ailleurs longtemps discourir sur les avantages et les inconvénients pour le Droit d'utiliser ou non des termes du langage commun dans les textes juridiques.

${ }^{9}$ Voir sur l'ensemble de la question, L. Eck, « La plage maritime : aspects renouvelés d'un espace naturel protégé », Petites Affiches, 29 mai $2008, n^{\circ} 108$, p. 8.

10 JOAN Questions écrites du 18 septembre 2007 : Réponse à Bernard Brochand, député des Alpes-Maritimes (p.5674).

${ }^{11}$ CE, Section, 30 mai 1975, req. n`83245, Dme Gozzoli, Lebon p. 325 ; AJDA 1975, p. 360, chron. M. Franc et M. Boyon : « Cons. que la parcelle dont s'agit ne fait pas partie du domaine public maritime, mais qu'il résulte de l'instruction que la partie de la plage où elle située est affectée au public et fait l'objet d'un entretien dans des conditions telles qu'elle doit être regardée comme bénéficiant d'un aménagement spécial à cet effet ; que dès lors, cette parcelle fait partie du domaine public communal » (soulignés par nos soins).

12 i.e. la partie du littoral située entre les niveaux connus des plus hautes et des plus basses mers dans la logique de la jurisprudence du Conseil d’Etat Kreitmann du 12 octobre 1973.
} 
comprend aussi des surfaces très importantes non seulement au titre de la dépression d'arrière-plage, mais aussi à l'avant-plage, ou haut de plage $\left({ }^{13}\right)$.

De même, il n'est pas certain que ces hauts de plage doivent être totalement ou partiellement recouverts par les flots pour être intégrés à la plage au sens du droit de l'urbanisme. Dans leur rapport de 2009, le Conseil général de l'environnement et du développement durable et l'inspection générale de l'administration, se réfèrent d'ailleurs à cette définition ministérielle en précisant que "sur les côtes à marées marquées, la plage est composée de deux parties partiellement distinctes : d'une part, l'estran, qui est à dimension variable, et d'autre part, une partie " sèche " qui se trouve au-dessus du niveau moyen des hautes eaux, mais qui reçoit les embruns et qui peut être submergée lors des très grandes marées ou lors de tempêtes $"\left({ }^{14}\right)$. Si on laisse de côté le critère des projections d'embruns dont on sait qu'elles dépassent allègrement les limites de la plage, il reste toujours même si c'est de manière exceptionnelle une exigence de submersion marine.

Par ailleurs, tout aussi classiquement, le rattachement des plages à la propriété publique apparaît aller de soi alors même qu'une partie des hauts de plage peut parfaitement appartenir à un propriétaire privé soit parce qu'ils ont été cédés par les communes, soit parce que la plage " physique " s'est étendue à des terrains privés notamment du fait de l'évolution des paysages dunaires. On notera également que certaines plages ont été concédées à des entreprises privées à des fins industrielles notamment pour l'exploitation saline.

Il semble dès lors que la recherche d'une définition de la plage fondée sur des qualifications juridiques préexistantes est illusoire. La plage apparaît largement composée de terrains d'importance inégale selon les façades maritimes et la topologie locale, mais relevant soit d'une partie " humide " car plus ou moins régulièrement recouverte par la mer soit d'une partie "sèche ", qui peut parfaitement échapper entièrement à toute submersion marine même exceptionnelle. En aucun cas, nous semble-t-il, la nature juridique du terrain en cause ne devrait influer sur un éventuel rattachement à la plage en tant que notion autonome.

Outre la difficulté à définir la plage sur le terrain juridique, la seconde difficulté rencontrée tient évidemment à la multiplicité des règles juridiques susceptibles de s'appliquer aux diverses parties de la plage. Au contraire de ce qui a pu être envisagé pour la définition de la plage, les diverses qualifications juridiques applicables aux différentes parties de la plage " physique » restent d'une importance primordiale dès lors qu'elles vont conditionner le régime juridique applicable. De fait, la question du droit applicable à la plage est d'une indéniable complexité. Celle-ci résulte de la multiplicité des propriétaires possibles de ce qu'on peut considérer comme une plage (Etat, communes, EPCI, propriétaires privés, gestionnaires des ports, titulaires d'autorisations diverses et variées) $\left({ }^{15}\right)$ et de la multiplication des statuts juridiques de ces terrains (domanialité publique, domanialité privée, propriété privée).

En outre, sur les mêmes terrains, coexistent parfois des régimes juridiques d'origines fort diverses $\left({ }^{16}\right)$. Ainsi, outre le droit de l'urbanisme, peuvent s'appliquer les règles générales de protection de la domanialité publique, mais également les règles spécifiques s'appliquant aux terrains militaires ou bien certaines servitudes administratives, comme la servitude de passage des piétons sur le littoral. Vont également s'imposer des règles de la police administrative, du droit des services publics, du droit portuaire ou du droit du tourisme. Le droit de l'urbanisme n'est donc que l'un des fondements possibles de la protection des plages. Même si certaines dispositions du droit de l'urbanisme sont indéniablement d'utilité courante (on pensera notamment au standard d'espace remarquable ou à

\footnotetext{
${ }^{13}$ Voir C. Maugüe, op. cit. et, pour une analyse du littoral du delta du Rhône, S. Suez et F. Sabatier, " Eléments de réflexion pour une gestion plus cohérente d'un système antropisé : exemple du littoral du delta du Rhône », Revue de géographie de Lyon, Vol.74, $\mathrm{n}^{\circ} 1,1999, \mathrm{p} .9$

${ }^{14}$ CGEDD et IGA, op. cit, p. 12.

${ }^{15}$ On pourra lire avec intérêt sur cette question (et cette difficulté) les remarques de scientifiques dont les recherches portent sur le littoral : S. Suanez et F. Sabatier, « Eléments de réflexion pour une gestion plus cohérencete d'un système anthropisé : exemple du littoral du delata du Rhône », op. cit., pp. 7-25.

${ }^{16}$ Sur cette question, voir notamment L. Bordereaux, « L'estran et son droit. - A propos du cœur du domaine public maritime naturel », Droit administratif $n^{\circ} 3$, mars 2012, étude 6.
} 
la bande de 100m), à la diversité des régimes de propriété correspondent des règles d'utilisation fort différentes. Ainsi, de manière courante, le droit de l'urbanisme se combine et s'associe à d'autres règles plus ou moins indépendantes au sens juridique du terme $\left({ }^{17}\right)$.

La question même du champ d'application du droit de l'urbanisme sur la plage est loin d'être aisée. Par exemple, les constructions sur les plages relevant du domaine publique maritime ne sont pas exonérées du respect des procédures et règles de fond du droit de l'urbanisme. Ainsi, conformément à l'article R. 431-13 du code de l'urbanisme, "lorsque le projet de construction porte sur une dépendance du domaine public, le dossier joint à la demande de permis de construire comporte une pièce exprimant l'accord du gestionnaire du domaine pour engager la procédure d'autorisation d'occupation temporaire du domaine public ". L'article L.421-5 du code de l'urbanisme ne dispense en effet d'autorisation de construire que les projets implantés "sur le domaine public immergé au-delà de la laisse de la basse mer $»\left({ }^{18}\right)$.

Cependant le droit de l'urbanisme n'a pas vocation à s'appliquer à l'ensemble des actes de gestion de ces dépendances. Ainsi, dans la fameuse affaire de la plage de Pampelonne, le Conseil d'Etat a certes confirmé l'illégalité d'un avenant à la concession de plage $\left({ }^{19}\right)$ mais, contrairement aux conclusions de son commissaire du gouvernement, sur le fondement du l'article L.321-5 du code de l'environnement (issu de la l'article 25 de la loi littoral) et non, comme le préconisait son commissaire du gouvernement, en inscrivant cette décision dans le champ de l'article L.146-6 du code de l'urbanisme $\left({ }^{20}\right)$. Autre exemple de la difficulté à déterminer les effets potentiels du droit de l'urbanisme sur le niveau de protection de plages avec le décret du 26 mai 2006 relatif aux concessions de plage $\left({ }^{21}\right)$. Pris sur le fondement de l'article L. 321-9 du code de l'environnement il pouvait apparaître comme indépendant du droit de l'urbanisme. Pourtant les interactions sont bien plus nombreuses qu'il n'y paraît de prime abord. Ainsi, le décret fait lui-même à plusieurs reprises référence à la notion d'espace remarquable au sens de l'article L. 146-6 du code de l'urbanisme $\left({ }^{22}\right)$. Pour autant, doit-il être considéré comme la loi spéciale dérogeant à la loi générale que serait l'article

\footnotetext{
${ }^{17}$ Cette question de la combinaison entre règles d'origine différente est d'ailleurs souvent une source de complexité. Si comme un auteur le notait «À cet égard, il convient d'observer la convergence entre les conditions relatives aux aménagements légers et le régime d'occupation des plages. Celui-ci prévoit que les équipements ou installations réalisés sur les plages doivent présenter un caractère strictement démontable, et interdit tout ancrage au sol. II prévoit également le retour, en fin de concession, à l'état initial du site, et l'obligation pour le concessionnaire de remettre en état les lieux ", les difficultés restent nombreuses. Ainsi alors que l'article R. 2124-14 prévoit que « Seuls sont permis sur une plage les équipements et installations démontables ou transportables ne présentant aucun élément de nature à les ancrer durablement au sol et dont l'importance et le coût sont compatibles avec la vocation du domaine et sa durée d'occupation. (...) Les installations autorisées sont déterminées en fonction de la situation et de la fréquentation de la plage ainsi que du niveau des services offerts dans le proche environnement. ", l'article L. 146-4-III permet la réalisation de "constructions ou installations nécessaires à des services publics ou à des activités économiques exigeant la proximité immédiate de l'eau » dans la bande littorale de 100m et l'article L. 146-6 des " aménagements légers » dans les espaces remarquables.

${ }^{18}$ L'article R. 421-8-1 dispose ainsi : "En application du e de l'article L.421-5, sont dispensées de toute formalité au titre du présent code, en raison de leur nature et de leur implantation sur le domaine public maritime immergé au-delà de la laisse de la basse mer, les installations de production d'électricité à partir de sources d'énergie renouvelable, y compris leurs ouvrages de raccordement aux réseaux publics d'électricité, notamment les éoliennes, les hydroliennes, les installations houlomotrices et marémotrices ainsi que celles utilisant l'énergie thermique des mers. "

${ }^{19}$ CE, 13 nov. 2002, Cne de Ramatuelle, AJDA 2003, p. 337, note J. Morand-Deviller ; BJDU 2002, p. 344, concl. D. Piveteau.

${ }^{20}$ Voir notamment sur cette question, J. Morand-Deviller, Espaces remarquables et aménagement des plages, AJDA, 2003, p. 337

${ }^{21}$ Désormais codifié aux articles R. 2124-13 et suivants du code général de la propriété des personnes publiques.

${ }^{22}$ Article R. 2124-16 du CG3P : "Seuls sont permis sur une plage les équipements et installations démontables ou transportables ne présentant aucun élément de nature à les ancrer durablement au sol et dont l'importance et le coût sont compatibles avec la vocation du domaine et sa durée d'occupation. Les équipements et installations implantés doivent être conçus de manière à permettre, en fin de concession, un retour du site à l'état initial. Leur localisation et leur aspect doivent respecter le caractère des sites et ne pas porter atteinte aux milieux naturels. Toutefois, les installations sanitaires publiques et les postes de sécurité peuvent donner lieu à des implantations fixes, sauf dans un espace remarquable au sens de l'article L. 146-6 du code de l'urbanisme. " Article R. 2124-19 du CG3P : " Les concessionnaires qui ont reçu du préfet l'agrément prévu à l'article R. 2124-18 délivrent, au cas par cas et après avis conforme du préfet, des autorisations annuelles spéciales permettant le maintien sur la plage, en dehors de
} 
L. 146-6 en dehors des cas où il y fait expressément référence ? On sait bien que la distinction entre loi générale et loi spéciale n'est simple que tant qu'on a pas en faire application. Elle peut devenir alors une grande source de perplexité et d'interrogations. Dans le cas qui nous intéresse, la logique comme le droit conduisent à imposer l'application conjointe du droit de l'environnement, du droit de l'urbanisme et de la loi Littoral.

De même, dans sa décision du 29 mars 2010, Depalle et Brosset c/ France, La Cour européenne des droits de l'homme montre bien non seulement la difficulté à opérer une qualification des contraintes imposées sur ce territoire mais aussi l'importance juridique du fondement d'une telle ingérerence aux éventuelles propriétés privées concernées. C'est très clairement la finalité environnementale, telle qu'elle affirmée par la loi Littoral, qui permet à la Cour de considérer de telles ingérences comme conformes à la Convention et non le régime de la domanialité publique susceptible également de s'y appliquer. $\left({ }^{23}\right)$

Ceci étant précisé, quels sont les types de constructions ou d'installations susceptibles de porter atteinte à la protection des plages telle qu'elle peut être assurée par le droit de l'urbanisme? En réalité elles sont de trois types qui, si elles ne présentent pas nécessairement de différences notables en matière de graduation de l'atteinte, reposent sur des logiques qu'on ne saurait assimiler. Tout d'abord il y a soit des constructions relevant de phénomènes que l'on qualifie habituellement de " cabanisation $"\left({ }^{24}\right)$, soit des constructions neuves ou rénovations situées sur les hauts de plage. Par ailleurs on trouve des constructions d'intérêt général qui relèvent des aménagements nécessaires ou pour le moins utiles à la fréquentation touristique de ces plages (postes de secours, sanitaires, aménagement pour les personnes à mobilité réduite, abris côtiers, parcs à bateaux...). Enfin on rencontre sur les plages des installations liées à des exploitations à caractère marchand (club de loisirs, boîtes de nuit et surtout restaurants de plage). C'est sans doute le développement sur la plage ou en bordure de la plage de ces activités commerciales dont certaines ont même une renommée internationale ainsi que la publicité donnée aux fameuses "paillotes » méditerranéennes qui ont conduit les pouvoirs publics à prendre conscience de l'atteinte - ou pour le moins des risques d'atteintes - à ces zones souvent fragiles et pourtant surfréquentées pendant la période estivale. Mais c'est également en raison de la nature marchande de ces activités commerciales que les dispositifs de protection ont été mis en cause ; l'emploi, le tourisme et le développement économique étant souvent et opportunément mis au service d'intérêts parfois plus financiers...et personnels.

$C^{\prime}$ est donc à la lumière de la diversité de ces règles juridiques mais aussi de la finalité sociale que l'on attribue à plage qu'il convient d'examiner et d'apprécier l'ensemble des dispositifs de protection des plages par le droit de l'urbanisme aussi bien par le biais de règles d'insconstructibilité qui lui sont propres que par l'utilisation des documents d'urbanisme prévisionnel.

\section{La protection renforcée de la plage par les règles d’inconstructibilité}

\footnotetext{
la période définie dans la concession, des établissements de plage démontables ou transportables situés en dehors d'un espace classé remarquable au sens de l'article L. 146-6 du code de l'urbanisme et qui remplissent les conditions suivantes (...) "

Article R. 2124-26 du CG3P : "Lorsque le projet est situé dans un espace remarquable au sens de l'article L. 146-6 du code de I'urbanisme, il ne peut être autorisé qu'après avis de la commission départementale compétente en matière de nature, paysages et sites."

${ }^{23}$ Voir sur cette très intéressante question, S. Manson, « De Pen er men à Strasbourg : le domaine public maritime français au miroir du droit européen des biens ॥, RDP n5-2010, pp 1451-1478.

${ }^{24}$ Sans être une exclusivité méditerranéenne, c'est un phénomène que l'on retrouve usuellement par exemple entre Marseille et Montpellier.
} 
Issue pour l'essentiel de la loi n86-2 du 3 janvier 1986, dite « Loi Littoral » $\left({ }^{25}\right)$, la protection spécifique dont bénéficie la plage du littoral s'exprime principalement par trois mécanismes fondamentaux $\left({ }^{26}\right)$ dont l'obet est de limiter ou d'interdire la réalisation de constructions ou d'aménagements sur la plage ou aux abords de celle-ci : la préservation des espaces remarquables, l'inconstructibilité dans la bande littorale de 100 mètres et, enfin, les restrictions à la réalisation des routes en bord de plage. Ces différentes protections bénéficient au demeurant d'un effet cumulatif. En effet, sans doute afin d'en maximiser les effets, le juge administratif n'hésite pas à appliquer sur la même bande littorale plusieurs de ces protections.

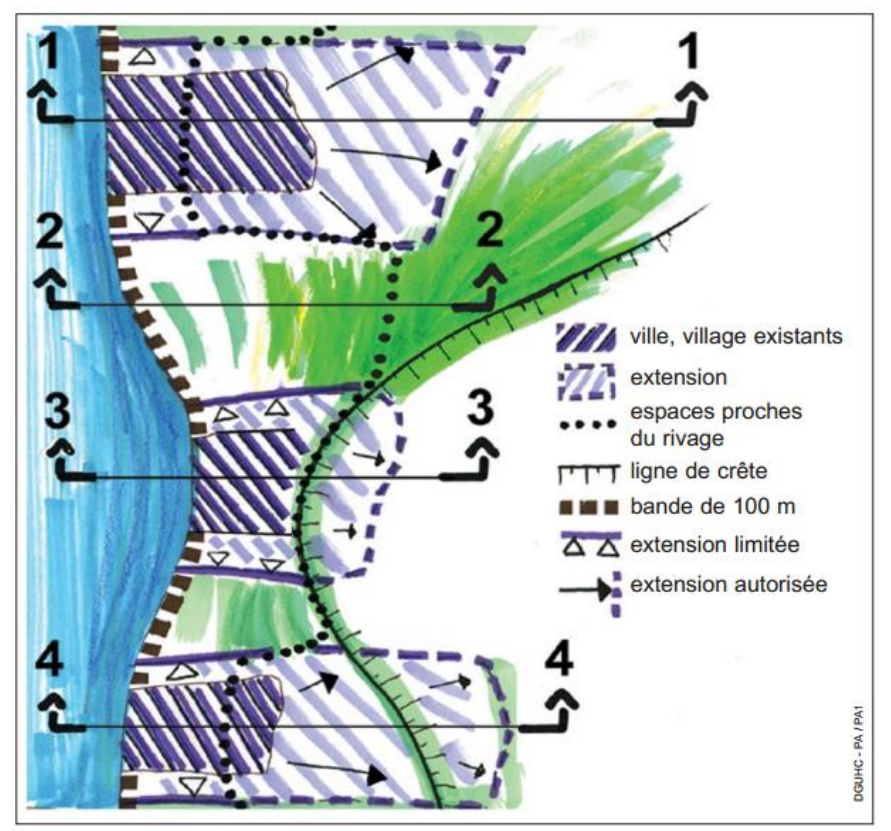

Source : Ministère chargé de l'Ecologie, du Développement durable et de l'Energie, Planifier l'aménagement, la protection et la mise en valeur du littoral, Juillet 2006, http://www.developpement-durable.gouv.fr/IMG/pdf/DGALN_Littoral_3 cle72d5d6.pdf, p.26, consulté le 10 septembre 2013.

\section{A. La protection de la plage en tant qu'espace, site, paysage remarquable ou caractéristique}

Aux termes de l'article L. 146-6 du code de l'urbanisme tel qu'il résulte de l'article 25 de la loi n²013-312 du 15 avril 2013 «Les documents et décisions relatifs à la vocation des zones ou à l'occupation et à l'utilisation des sols préservent les espaces terrestres et marins, sites et paysages remarquables ou caractéristiques du patrimoine naturel et culturel du littoral, et les milieux nécessaires au maintien des équilibres biologiques. (...) Toutefois, des aménagements légers peuvent y être implantés lorsqu'ils sont nécessaires à leur gestion, à leur mise en valeur notamment économique ou, le cas échéant, à leur ouverture au public. (...) Peuvent être également autorisées les canalisations du réseau public de transport ou de distribution d'électricité visant à promouvoir l'utilisation des énergies renouvelables. Les techniques utilisées pour la réalisation de ces ouvrages sont souterraines et toujours celles de moindre impact environnemental. L'approbation des projets de construction des ouvrages, mentionnée au $1^{\circ}$ de l'article L. 323-11 du code de l'énergie, est refusée si les canalisations sont de nature à porter atteinte à l'environnement ou aux sites et paysages remarquables. Leur réalisation est soumise à enquête publique réalisée conformément au chapitre III du titre II du livre ler du code de l'environnement. En outre, la réalisation de travaux ayant pour objet la conservation ou la protection de ces espaces et milieux peut être admise, après enquête publique réalisée conformément au chapitre III du titre II du livre ler du code de l'environnement."

L'article L. 146-6 du Code de l'urbanisme est indubitablement devenu au fil des évolutions de la jurisprudence l'un des outils majeurs de la protection des plages. Sans doute, l'affaire dite de la Plage de Pampelonne $\left({ }^{27}\right)$ a beaucoup fait

\footnotetext{
${ }^{25}$ Même si certains mécanismes de protection apparaissaient déjà dans la Directive d'aménagement national du 25 août 1979 , dite « Directive d'Ornano » comme la préservation de la bande de 100 mètres.

${ }^{26}$ Les règles de l'article L. 146-4-II relatives à l'extension limitée des espaces proches du rivage n'ont sans doute pas pour la protection des plages le même intérêt que pour les espaces un peu plus éloignées du rivage. La définition même de " l'espace proche du rivage » renvoie d'ailleurs souvent à un critère de visibilité de la plage et non de situation sur la plage.

${ }^{27}$ CE, 13 nov. 2002, Cne de Ramatuelle, op. cit.

JPF - Colloque du 9 avril 2013 : "Sous Ia plage, le droit "

Centre de Droit et de Politique Comparés CDPC Jean-CIaude Escarras
} 
pour sa renommée, mais la jurisprudence ne saurait être artificiellement limitée à cet arrêt. Une jurisprudence riche, confortée par les dispositions réglementaires du code de l'urbanisme, vient directement étayer l'applicabilité de cet article aux plages.

Ce n'est d'ailleurs pas seulement un mécanisme complémentaire de l'article L. 146-4-III. En toute logique, et pour peu que les deux moyens correspondants soient soulevés comme cela arrive fréquemment, le juge doit donc examiner celui tiré de la violation des dispositions de l'article L. 146-6 avant celui tiré de la méconnaissance de celles de l'article L. 146-4-III ( $\left.{ }^{28}\right)$. C'est donc en premier lieu en tant qu'espace naturel proche du rivage que les plages font l'objet d'une protection renforcée par le droit de l'urbanisme.

L'article R. 146-1 ne laisse aucun doute sur l'applicabilité de l'article L. 146-6 aux plages puisqu'il prévoit notamment au sein d'une liste des espaces susceptibles d'être protégés à ce titre un a) qui vise précisément " Les dunes, les landes côtières, les plages et les lidos, les estrans, les falaises et les abords de celles-ci ». Mais, surtout, au regard de multiples exemples jurisprudentiels, le juge administratif semble avoir fait des plages un lieu d'application privilégiée du standard d'espace remarquable non seulement pour les hauts de plage, mais aussi sur le domaine public maritime (DPM). Certes la plage (au sens le plus large du terme) n'est pas en elle-même un espace remarquable. Cependant, il n'est pas nécessaire qu'elle ait fait préalablement l'objet d'une protection particulière au titre par exemple des sites inscrits ou classés $\left({ }^{29}\right)$.

Néanmoins, cette protection a pu apparaître altérer par la combinaison de l'article L. 146-6 avec les dispositions particulières applicables aux plages concédées. Sans doute fondés sur l'idée que la concession traduit la volonté d'un nouvel équilibre entre protection et aménagement, un certain nombre de sous-traités de concession ont prévu la réalisation de constructions ou installations afférentes à l'exploitation de la concession. Cependant, le traité comme le sous-traité de concession ne sauraient exempter le concessionnaire du respect de cette disposition. Est ainsi illégale la concession de plage permettant la construction de bâtiments sur le domaine public maritime dès lors que la plage est qualifiée d'espace remarquable. II résulte ainsi de la combinaison des règles applicables aux concession (et donc sur le DPM) et de l'article 146-6 que seules peuvent être autorisées sur le DPM des installations à caractère " démontable » et, en dehors du DPM, des installations à caractère " réversible ».

\footnotetext{
${ }^{28}$ P. Fraisseix, "Le juge administratif et l'article L. 146 du code de l'urbanisme : onze années d'interprétation prétorienne », RFDA 1998, p.834 «En réalité cet article bénéficie d'une sorte d'autorité supérieure en ce sens qu'en matière d'extension de l'urbanisation des "espaces proches " du littoral comme en matière de création de route, l'autorité juridictionnelle contrôlera toujours si l'espace en cause ne constitue pas un espace "remarquable " avant même que d'examiner si l'opération projetée respecte successivement les articles L. 146-4-II et L. 146-7. »

${ }^{29}$ A l'inverse les plages naturelles inscrites ou classées au titre de la loi loi du 2 mai 1930 portant réorganisation de la protection de monuments naturels et des sites à caractère artistique, historique, scientifique, légendaire ou pittoresque sont présumées être des espaces remarquables (CE, 20 octobre 1995, Cne de Saint-Jean Cap-Ferrat, Rec p.282 ou CE, 13 nov. 2002, Cne de Ramatuelle, op. cit.). La rédaction de l'arrêt Cne de Ramatuelle (op.cit.) est à ce titre significatif : " Considérant qu'il ressort des pièces du dossier soumis aux juges du fond que les parcelles concernées par les permis de construire litigieux sont situées sur la plage de Pampelonne, elle-même située dans la presqu'île de Saint-Tropez; qu'ainsi que la cour l'a constaté cette presqu'île est un site inscrit au titre de la loi du 2 mai 1930 ; Considérant que, pour déterminer si la plage de Pampelonne constituait une partie naturelle de ce site inscrit, la cour administrative d'appel a, contrairement à ce que soutiennent les requérants, recherché, tant sur la plage elle-même que dans son environnement immédiat, l'existence d'un certain degré d'urbanisation ou d'autres altérations liées à l'activité humaine ; qu'en estimant, au terme d'une appréciation souveraine, que l'existence d'un lotissement situé à l'arrière de la plage et de quelques bâtiments sur la plage elle-même ne pouvait suffire à ôter à cette dernière son caractère naturel, la cour administrative d'appel n'a pas dénaturé les faits de l'espèce ; que, sans commettre d'erreur de droit, elle a pu déduire de ce constat, dès lors que les parties naturelles des sites inscrits sont présumées constituer des sites ou paysages remarquables et que cette qualification présumée n'était en l'espèce pas contestée devant elle, que la plage de Pampelonne et son cordon dunaire constituaient l'un des espaces remarquables dont le législateur a entendu assurer la préservation; "

Comme le signale R. Hostiou, les auteurs du décret n²004-310 du 29 mars 2004 modifiant l'article R. 146-2 avaient envisagé de remettre en cause cette présomption avant d'y renoncer sans doute par crainte des réactions (R. Hostiou, « Espaces remarquables du littoral : le changement dans la continuité ", AJDA 2005, p.370).
} 
Si le principe de l'applicabilité du standard d'espace remarquable à la plage n'a guère fait de doutes, en revanche le régime d'exceptions a donné lieu à un contentieux abondant. En effet, en autorisant dans les espaces remarquables la réalisation d'aménagements légers, le législateur a fait naître beaucoup d'incompréhension aussi bien chez les protecteurs de l'environnement que chez les élus, les aménageurs ou les professionnels du tourisme. Malgré une modification de sa rédaction par le décret n² 2004 -310 du 29 mars 2004, l'article R. 146-2 a continué d'engendrer un contentieux important. Comme souvent avec de tels textes, les précautions rédactionnelles n'ont été que de peu d'influence sur la compréhension du texte tellement étaient fortes les attentes contradictoires $\left({ }^{30}\right)$.

L'article R. 146-2 prévoit en effet un certain nombre d'hypothèses et de conditions $\left({ }^{31}\right)$ permettant à la fois de caractériser l'aménagement léger au sens de ce texte et d'en déterminer le champ d'utlisation. Tout d'abord, comme le rappelle la circulaire de 2005, "le caractère léger s'appréciera au regard de la hauteur, du volume, du rapport hauteur / emprise au sol, de la taille de la construction, notamment au regard des dimensions du site. En particulier, l'aménagement devra conserver des proportions raisonnables et on appréciera son incidence sur l'environnement. " De même ces aménagements légers "doivent être conçus de manière à permettre un retour du site à l'état naturel. " Ensuite l'article R. 146-2 vise quatre hypothèses : les aménagements nécessaires à l'accueil du public (chemins, objets mobiliers, postes d'observation, sanitaires, postes de secours, aires de stationnement), la réfection de bâtiments existants et extensions limitées nécessaires à des activités économiques (notamment les activités nécessitant la proximité immédiate de l'eau) et les aménagements nécessaires à la remise en état d'éléments de patrimoine bâti. Sans rentrer dans les détails d'une jurisprudence particulièrement fournie, les articles L. 146-6 et R. 146-2 ne permettent donc pas non plus la réalisation des installations même légères nécessaires aux activtés de restauration $\left.{ }^{(32}\right)$, sportives ou de jeux $\left({ }^{33}\right)$.

Par le caractère strict de son interprétation, le juge a ainsi fait de l'article L. 146-6 le principal fondement de la protection directe de la plage au titre du droit de l'urbanisme. "Par les conséquences qu'elle emporte, une quasiinconstructibilité de fait, la qualification d'espace ou de site ou de paysage remarquable ou caractéristique, au titre de I'article L. 146-6 du Code de l'urbanisme, constitue certainement la protection la plus efficace des espaces littoraux autres que ceux situés dans la bande des 100 mètres $"\left({ }^{34}\right)$.

\footnotetext{
${ }^{30}$ La circulaire $n^{\circ} 2005-57$ du 15 septembre 2005 relative aux nouvelles dispositions prévues par le décret $n^{\circ} 2004-310$ du 29 mars 2004 relatif aux espaces remarquables du littoral et modifiant le code de l'urbanisme a bien essayé de systématiser les aménagements ainsi autorisés mais sans y parvenir pleinement.

${ }^{31}$ L'article R. 146-2 dispose que : «En application du deuxième alinéa de l'article L. 146-6, peuvent être implantés dans les espaces et milieux mentionnés à cet article, après enquête publique dans les cas prévus par les articles R. 123-1à R. 123-33 du code de l'environnement, les aménagements légers suivants, à condition que leur localisation et leur aspect ne dénaturent pas le caractère des sites, ne compromettent pas leur qualité architecturale et paysagère et ne portent pas atteinte à la préservation des milieux :

a) Lorsqu'ils sont nécessaires à la gestion ou à l'ouverture au public de ces espaces ou milieux, les cheminements piétonniers et cyclables et les sentes équestres ni cimentés, ni bitumés, les objets mobiliers destinés à l'accueil ou à l'information du public, les postes d'observation de la faune ainsi que les équipements démontables liés à l'hygiène et à la sécurité tels que les sanitaires et les postes de secours lorsque leur localisation dans ces espaces est rendue indispensable par l'importance de la fréquentation du public; b) Les aires de stationnement indispensables à la maîtrise de la fréquentation automobile et à la prévention de la dégradation de ces espaces par la résorption du stationnement irrégulier, sans qu'il en résulte un accroissement des capacités effectives de stationnement, à condition que ces aires ne soient ni cimentées ni bitumées et qu'aucune autre implantation ne soit possible ; c) La réfection des bâtiments existants et l'extension limitée des bâtiments et installations nécessaires à l'exercice d'activités économiques; d) A l'exclusion de toute forme d'hébergement et à condition qu'ils soient en harmonie avec le site et les constructions existantes:

-les aménagements nécessaires à l'exercice des activités agricoles, pastorales et forestières ne créant pas plus de 50 mètres carrés de surface de plancher; -dans les zones de pêche, de cultures marines ou lacustres, de conchyliculture, de saliculture et d'élevage d'ovins de prés salés, les constructions et aménagements exigeant la proximité immédiate de l'eau liés aux activités traditionnellement implantées dans ces zones, à la condition que leur localisation soit rendue indispensable par des nécessités techniques; e) Les aménagements nécessaires à la gestion et à la remise en état d'éléments de patrimoine bâti reconnus par un classement au titre de la loi du 31 décembre 1913 ou localisés dans un site inscrit ou classé au titre des articles L. 341-1 et L. 341-2 du code de l'environnement. Les aménagements mentionnés aux a, $b$ et $d$ du présent article doivent être conçus de manière à permettre un retour du site à l'état naturel. "

32 CAA Marseille, 8 avr. 2005, Cne Croix-Valmer, nº1MA01755 et 01 MA01971.

${ }^{33}$ CE, 20 oct. 1995, Cne de Saint-Jean-Cap-Ferrat, Rec. Lebon, tabl., p. 1072.

${ }^{34}$ L. Benoit, E. Coënt-Bochard, Pierre Larroumec, Conseillers aux cours administratives d'appel de Marseille, Nantes et Bordeaux, "La loi Littoral devant les cours administratives d'appel », AJDA 2002, p. 600. Les auteurs soulignent, de manière très intéressante,
} 


\section{B. La protection de la plage située dans la bande littorale des 100 mètres.}

Aux termes de l'article L. 146-4-III du code de l'urbanisme : "En dehors des espaces urbanisés, les constructions ou installations sont interdites sur une bande littorale de cent mètres à compter de la limite haute du rivage ou des plus hautes eaux pour les plans d'eau intérieurs désignés à l'article 2 de la loi $n^{\circ} 86-2$ du 3 janvier 1986 précitée. (...) Le plan local d'urbanisme peut porter la largeur de la bande littorale visée au premier alinéa du présent paragraphe à plus de cent mètres, lorsque des motifs liés à la sensibilité des milieux ou à l'érosion des côtes le justifient. »

L'article L. 146-4-III apporte immédiatement une limitation, somme toute théoriquement mineure mais source de nombreux contentieux, en prévoyant que "cette interdiction ne s'applique pas aux constructions ou installations nécessaires à des services publics ou à des activités économiques exigeant la proximité immédiate de l'eau, et notamment aux ouvrages de raccordement aux réseaux publics de transport ou de distribution d'électricité des installations marines utilisant les énergies renouvelables. Leur réalisation est toutefois soumise à enquête publique réalisée conformément au chapitre III du titre II du livre ler du code de l'environnement. Un décret en Conseil d'Etat fixe les conditions de réalisation des ouvrages nécessaires au raccordement aux réseaux publics de transport ou de distribution d'électricité des installations marines utilisant les énergies renouvelables. Les techniques utilisées pour la réalisation de ces raccordements sont souterraines et toujours celles de moindre impact environnemental.»

Du fait de leur localisation à proximité immédiate du rivage, les plages sont très directement concernées par ce dispositif. Sur la façade méditerranéenne, notamment, la très faible largeur de la bande littorale (au sens géomorphique) ainsi que les phénomènes de recul du trait de côte conduisent à une intégration presque systématique de la surface totale des plages dans le champ d'application de ce texte. Elles bénéficient donc, de facto, d'une protection renforcée assurée par l'article L. 146-4-III. Toutefois, en pratique, cette règle connaît plusieurs problèmes d'application soit en raison de la relative indétermination des termes utilisés soit en raison des conditions et des exceptions prévues par cette disposition elle-même.

Les interrogations soulevées à l'occasion de la mise en œuvre de cette règle sont de deux ordres. Tout d'abord, contrairement à ce que son intitulé courant « bande littorale des $100 \mathrm{~m}$ » pourrait laisser croire, la limite supérieure de cette zone protégée fait l'objet d'une très large indétermination. Elle est ainsi très rarement matérialisée dans les documents d'urbanisme communaux ${ }^{(35}$ ) et elle est loin d'être fixe et précise. Déterminée à partir de la limite haute du rivage, elle connait les mêmes évolutions tendancielles que la limite du DPM qui utilise un critère de délimitation identique $\left({ }^{36}\right)$. Comme l'a montré le Conseil constitutionnel dans sa décision n²013-316-QPC du 24 mai 2013 relative à la délimitation du DPM, non seulement la consistance du DPM peut notablement varier mais, surtout, il n'est pas possible d'ignorer les conséquences de la progression du rivage de la mer. Dès lors, il convient alors d'assurer une protection (certes encore très relative) des « droits acquis » du propriétaire ainsi affecté par la remontée de la ligne de côte $\left({ }^{37}\right)$.

En outre, à cette relative indétermination des limites de cette bande littorale de $100 \mathrm{~m}$, il convient d'ajouter les nombreuses interrogations qu'ont pu faire naître les exceptions à l'interdiction des constructions ou installations dans cette bande de $100 \mathrm{~m}$. En premier lieu, cette interdiction ne vaut, conformément à l'article L. 146-4-III qu' "En dehors des espaces urbanisés ". Si cette notion est assez classiquement utilisée en droit de l'urbanisme (une dizaine

qu'avant la mise en œuvre de l'article L. 600-4-1 lorsque la juridiction compétente était saisie à la fois d'un moyen fondé sur l'article L.146-6 et d'un moyen fondé sur l'article L.146-4 « le juge (devait) donc examiner celui tiré de la violation des dispositions de l'article L. 146-6 avant celui tiré de la méconnaissance de celles de l'article L. 146-4 ».

${ }^{35}$ Voir seconde partie.

${ }^{36}$ Voir sur cette question les multiples développements doctrinaux portant à la fois sur la logique de la jurisprudence Kreitman et sur l'application de l'article L. 2111-4 du code général de la propriété publique.

${ }^{37}$ Le Conseil précise ainsi, dans le considérant $n^{\circ} 8$, " que, dans ces conditions, la garantie des droits du propriétaire riverain de la mer ayant élevé une digue à la mer ne serait pas assurée s'il était forcé de la détruire à ses frais en raison de l'évolution des limites du domaine public maritime naturel ». Il conviendra sans doute d'examiner les conséquences jurisprudentielles que le Conseil d'Etat pourrait tirer de cette réserve d'interprétation. A cet égard on pourra lire avec intérêt N. Foulquier, « La délimitation du domaine public maritime : I'expropriation inavouée «, RDI 2012, p.348 
d'occurrences dans le code de l'urbanisme), elle ne se caractérise pas nécessairement par sa précision même si elle apparaît à la source d'un contenbtieux important $\left({ }^{38}\right)$. Notion qui échappe pour l'instant à toute définition précise $\left({ }^{39}\right)$, elle subit fort logiquement dans son application l'influence des éléments purement factuels $\left({ }^{40}\right)$, ce qui évidemment renforce les difficutés d'interprétation $\left({ }^{41}\right)$.

En second lieu, cette interdiction stricte de toute construction ou installation dans la bande de $100 \mathrm{~m}$ ne s'applique pas " aux constructions ou installations nécessaires à des services publics ou à des activités économiques exigeant la proximité immédiate de l'eau " que le législateur a précisé de manière interprétative en y incluant notamment les " ouvrages de raccordement aux réseaux publics de transport ou de distribution d'électricité des installations marines utilisant les énergies renouvelables. Sans que ce soit véritablement une surprise, la jurisprudence a adopté une interprétation indéniablement porteuse d'une politique jurisprudentielle marquée par son caractère restrictif. Si des toilettes publiques, un local de secours, au nom d'impératifs de santé et de sécurité publiques $\left({ }^{42}\right)$, ou bien des activités d'aquaculture $\left({ }^{43}\right)$ ou de mareyage $\left({ }^{44}\right)$ ont pu apparaître comme satisfaisant à l'obligation de proximité immédiate de l'eau. A l'inverse un centre de thalassothérapie $\left({ }^{45}\right)$, un bar-restaurant $\left({ }^{46}\right)$ ou plus simplement un parking $\left({ }^{47}\right)$ n'ont pas été considérés comme nécessitant cette proximité. Si de telles solutions apparaissent éminemment liés aux faits des espèces, on ne peut pas ne pas y voir une volonté restrictive à l'égard des équipements " récréatifs » inhérents à la présence du public. Le juge distingue particulièrement les activités exigeant la proximité immédiate de l'eau (location

\footnotetext{
${ }^{38}$ Voir en ce sens, CGEDD, « Audit thématique sur l'application de la loi Littoral par les services de l’Etat », op. cit. p.68.

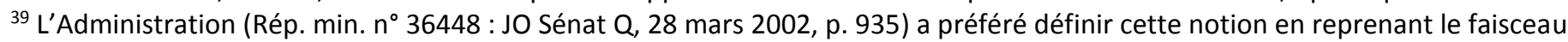
d'indices élaboré par le juge : "La notion d'espaces urbanisés au sens de l'article L. 146-4-III du code de l'urbanisme en dehors desquels une servitude d'inconstructibilité affecte une bande littorale de cent mètres, se distingue de la notion de zone bâtie. Le caractère urbanisé ou non d'un espace s'apprécie au regard notamment de la densité de construction (CE, 27 janvier 1997, Djerelian, req. $n^{\circ}$ 125842), de la desserte par des équipements collectifs du secteur environnant la parcelle supportant le projet de construction en cause (CE, 15 janvier 1997, Malfatto, req. $\left.n^{\circ} 125937\right)$, de la situation de cette parcelle à l'intérieur de cet espace, de sa plus ou moins grande proximité des habitations existantes (CE, 12 mais 1997, Ifana, req. $n^{\circ}$ 163352) ainsi que des divers obstacles physiques qui peuvent séparer cette parcelle des zones d'habitations existantes. Une espace urbanisé est nécessairement bâti tandis qu'une zone bâtie n'est pas nécessairement comprise dans un espace urbanisé. "

40 Voir en ce sens, L. Prieur, "La Loi Littoral et son contentieux administratif», https:// www.ouestcornouaille.com\%2Fbretagne sud finistere\%2Fla-loi-littoral-et-son-contentieux-

administratif\%2Farticle\%2F225\&ei=poRkUv2vOcLL0QXQ84HoBA\&usg=AFQjCNGjdqBP8D5neK5PcD9SZWsJDVChTw\&bvm=bv.549 34254,d.d2k., consulté le 10 septembre 2013.

${ }^{41}$ On se réfèrera notamment à CGEDD, "Audit thématique sur l'application de la loi Littoral par les services de l’Etat ", op. cit. pp.67-70. Les auteurs y montrent de manière très intéressante les difficultés rencontrées par les services de l'Etat lorsqu'il s'agit de qualifier un espace urbanisé littoral. Avec une circulaire mal rédigée et contestée par les services, un juge qui apprécie souverainement et in concreto cette notion, une administration déconcentrée qui manque de moyens, on a un recensement des principales difficultés rencontrées. Pour autant les propositions des auteurs du rapport ne sont pas non plus à l'abri de tout critique. En souhaitant une distinction entre les espaces urbanisés selon qu'il s'agit d'une agglomération, d'un village, d'un hameau ou d'un autre type d'espaces urbanisés, ils se retouvent en contradiction avec le Conseil d'Etat (CE, 22 février 2008, $n^{\circ} 280189$ ) qui considère qu'un " espace urbanisé au sens des dispositions du III de l'article L. 146-4 précité appartient, par nature, à une agglomération ou à à un village existant au sens du I du même article ".

${ }^{42}$ De manière générale, le Conseil d’Etat a considéré que "les impératifs de sécurité et de santé publique liés à une fréquentation estivale importante des plages peuvent requérir l'implantation d'installations nécessaires au public ", et que " dans le cas d'aménagement de constructions existantes, de telles installations peuvent être regardées comme nécessaires à des services publics exigeant la proximité immédiate de l'eau au sens du III de l'article L. 146-4 du code de l'urbanisme " (CE, 8 octobre 2008, $\left.\mathrm{n}^{\circ} 293469\right)$.

43 TA Nice, 6 juill. 1995, n 90-2198, Sté France-travaux.

44 TA Rennes, 24 mars 1994, Le Corre.

45 TA Nice, 17 déc. 1987, Mouvement niçois pour défense sites et patrimoine et a., RFDA 1990, p. 234, concl. N. Caldéraro.

${ }^{46}$ CAA Marseille, 19 sept. 2002, Préfet de Corse du sud, RJE. 2003, p. 497 ou CE, 9 oct. 1996, n 161555, Union départementale Vie et Nature 83.

${ }^{47} \mathrm{CE}, 10$ mai 1996, n 155169, Cne de Saint-Jorioz.
} 
de matériels nautiques par exemple), de celles comme la restauration ou l'hébergement qui ne font qu'accompagner l'activité touristique $\left({ }^{48}\right)$.

\section{La protection de la plage par des restrictions à la réalisation de routes sur le littoral}

En vertu de l'article L. 146-7 la réalisation de nouvelles routes à proximité des plages est subordonnée à des conditions restrictives auxquelles il ne peut être dérogé qu'en raison de contraintes liées à la configuration des lieux. Ainsi non seulement "Les nouvelles routes de transit sont localisées à une distance minimale de 2.000 mètres du rivage " (alinéa 2), mais de manière particulièrement protectrice « La création de nouvelles routes sur les plages, cordons lagunaires, dunes ou en corniche est interdite. Les nouvelles routes de desserte locale ne peuvent être établies sur le rivage, ni le longer. " (alinéa 3). Ces règles qui se conjuguent avec celles de l'article L. 146-6 $\left({ }^{49}\right.$ ) assurent un niveau supplémentaire élevé de protection de la plage. Au surplus, elles complètent les différentes règles relatives au stationnement des véhicules afin, en théorie, de faire de la plage un espace sans véhicules $\left({ }^{50}\right)$.

Deux types d'exceptions sont prévues à cette interdiction. Une première exception résulte de la configuration des lieux. L'article L. 146-7, dans son alinéa 5, prévoit en effet que " les dispositions des deuxième, troisième et quatrième alinéas ne s'appliquent pas en cas de contraintes liées à la configuration des lieux ou, le cas échéant, à l'insularité. La commission départementale compétente en matière de nature, de paysages et de sites est alors consultée sur l'impact de l'implantation de ces nouvelles routes sur la nature. En outre, l'aménagement des routes dans la bande littorale définie à l'article L. 146-4 est possible dans les espaces urbanisés ou lorsqu'elles sont nécessaires à des services publics ou à des activités économiques exigeant la proximité immédiate de l'eau. »

En second lieu, I'article L. 146-8 exclut de ce régime de protection « Les installations, constructions, aménagements de nouvelles routes et ouvrages nécessaires à la sécurité maritime et aérienne, à la défense nationale, à la sécurité civile et ceux nécessaires au fonctionnement des aérodromes et des services publics portuaires autres que les ports de plaisance ne sont pas soumis aux dispositions du présent chapitre lorsque leur localisation répond à une nécessité technique impérative. " Si un tel dispositif apparaît bien large, il répond très clairement à la volonté de permettre, en matière routière, la desserte des réalisations d'intérêt général dont l'Etat a la charge $\left({ }^{51}\right)$.

\footnotetext{
${ }^{48}$ Voir, par exemple, CAA Marseille, 12 avril 2012, U Levante, $\mathrm{n}^{\circ} 10 \mathrm{MA} 02237$ : " Considérant que le permis litigieux autorise l'aménagement d'un commerce de plage à structure démontable en bordure de plage à Cagnano, à moins de 100 mètres du rivage, dans un secteur non urbanisé ; qu'ainsi, le terrain d'assiette de la construction est situé en dehors des espaces urbanisés de la commune au sens des dispositions précitées, ce qui n'est d'ailleurs pas contesté ; qu'il s'ensuit que le maire ne pouvait autoriser le projet que si la construction était nécessaire à des services publics ou à des activités économiques exigeant la proximité immédiate de l'eau ; que si l'activité de location de matériels nautiques est une activité exigeant la proximité immédiate de l'eau, en revanche, l'activité de restauration, liée à l'exploitation d'un snack-grill et dissociable de l'activité de location de matériels nautiques, ne nécessite pas une telle localisation. "

${ }^{49}$ Sur la question de la complémentarité entre les protections assurées par les les articles L. 146-7 et L. 146-4, voir CE, 25 novembre

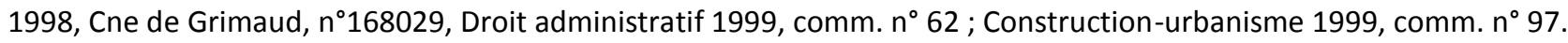

${ }^{50}$ On notera cependant que ce bannissement n'est pas total ainsi qu'en atteste les nombreux contentieux relatifs à l'Enduro du Touquet qui se déroule sur la plage après avoir longtemps utilisé les dunes. Voir par exemple, B. Bernabeu, « Le juge administratif et l'Enduro du Touquet ", JCP A n52, 26 décembre 2006, 1320.

${ }^{51}$ Par exemple, dans un arrêt du 29 décembre 1999, Epx Mautalent, $n^{\circ} 197720$, le Conseil d’Etat justifie ainsi la réalisation d’une telle route : "Considérant que le projet de contournement de l'agglomération cherbourgeoise par l'Est, destiné à relier le port de commerce de Cherbourg au réseau routier national, est devenu nécessaire au fonctionnement du service public portuaire, en raison du développement du trafic maritime à travers la Manche; que sa localisation à proximité du littoral, dans sa partie terminale, répond ainsi à la nécessité de desservir le port; qu'elle est en outre tributaire des contraintes résultant de l'urbanisation ; que dans ces conditions, les requérants ne sont pas fondés à soutenir que le décret attaqué aurait méconnu l'article L. 146-8 du code de I'urbanisme; que le moyen tiré de la violation de l'article L. 146-7 du même code ne peut en conséquence qu'être lui aussi écarté ;»
} 


\section{La protection en devenir de la plage par l'urbanisme prévisionnel}

Le rôle des documents d'urbanisme dans la protection des territoires littoraux et, plus spécifiquement des plages, est fondamental. Comme l'expliquait le rapporteur du projet de loi Littoral devant l'Assemblée nationale, ce texte " s'inscrit [...] dans une ligne nouvelle d'écriture du droit, dont les premiers termes apparaissent dans les directives [d'aménagement national] déjà évoquées. Il est donc légitime que la loi ne soit pas écrite en des termes déjà juridiques ou définis parfaitement et cela relève de sa nature. C'est aux décideurs locaux de traduire, dans des documents ou par les différentes autorisations délivrées, le droit applicable [...]. Le juridique n'intervient qu'après que les choix politiques ont été exercés » ${ }^{52}$ ). Or c'est un à travers les documents d'urbanisme, qui expriment les choix politiques globaux, que la loi Littoral prend tout son sens $\left({ }^{53}\right)$. Comme l'envisageait la circulaire $n^{\circ} 2006-31$ du 14 mars 2006 relative à l'application de la loi Littoral, des opérations d'aménagement qui, prévues au coup au coup ou dans un cadre trop restreint, seraient illégales, pourraient être autorisées dès lors qu'un document les a imaginées dans une politique globale d'équilibre entre le développement et la protection et à une échelle pertinente $\left({ }^{54}\right)$. II s'agit là d'un atout important de l'urbanisme prévisionnel qui, seul, permet d'envisager la protection et l'amènagement des plages dans le cadre d'un urbanisme durable eu égard à la triple menace qui pèse sur les plages : submersion marine, érosion du trait de côte et déplacement dunaire.

En même temps, cette fonction de l'urbanisme prévisionnel suscite encore bien des interrogations et des doutes $\left({ }^{55}\right)$. Elle reste d'ailleurs généralement bien en deçà des espérances $\left({ }^{56}\right)$ même si les instruments spécifiques ou plus généraux ne manquent pas.

\section{A. La plage dans les documents d'urbanisme spécifiques}

Les principaux documents d'urbanisme spécifiques que sont les Schémas de mise en valeur de la mer (SMVM) ou les schémas d'aménagement de la plage s'inscrivent dans une politique plus globale initiée à la fois par l'Etat et l'Union européenne. A ce titre, on ne peut que constater une nouvelle fois le mille-feuille normatif auquel est soumis le littoral.

\footnotetext{
52 L. Lacombe, Une législation pour le littoral, Pourquoi ? Pour quels objectifs ?, La loi Littoral, Économica, 1987, p. 17, cité par L. Prieur, "L'écriture des plans locaux d’urbanisme littoraux, Fiche 3 : La traduction de la loi littoral dans les PLU littoraux », op. cit., p. 2 .

${ }^{54}$ Voir, par exemple, CE., 27 juillet 2005, Comité de sauvegarde du port Vauban, Vieille-Ville et Antibes-Est req. no 264336 : « En faisant figurer le secteur dit des pétroliers à Antibes au nombre des espaces où le caractère limité de l'extension de l'urbanisation dans les espaces proches du rivage doit s'apprécier compte tenu de la capacité d'accueil de ces espaces et de l'intégration des opérations projetées dans l'environnement tout en en imposant d'apprécier l'extension de l'urbanisation qui résultera de ce classement au regard de l'agglomération dans son ensemble et compte tenu des autres orientations qu'elle définit par ailleurs, la DTA des Alpes-maritimes, avec laquelle les documents d'urbanisme applicables à ce secteur et les autorisations d'urbanisme qui y seront délivrées devront être, respectivement, compatibles et conformes, ne méconnaît pas les dispositions du II de l'article L. 146-4 du code de l'urbanisme. "

55 J. Daligaux, « La loi Littoral à l'épreuve des SCOT », Etudes foncières, juill.-août 2005, p. 33.

${ }^{56}$ L'administration elle-même accorde une place bien plus importante au volet normatif de la protection du littoral qu'à son volet prévisionnel. Par exemple, la circulaire n²005-57 du 15 septembre 2005 (op. cit.), ne consacre que quelques lignes au rôle des documents d'urbanisme : "L'article 1er du décret codifié à l'article R. 146-1 indique que "Lorsqu'ils identifient des espaces ou milieux relevant du présent article, les documents d'urbanisme précisent, le cas échéant, la nature des activités et catégories d'équipements nécessaires à leur gestion ou à leur mise en valeur notamment économique. "Le plan local d'urbanisme, peut, à l'article 2 du règlement, préciser que les aménagements et constructions nécessaires à ces activités ou équipements sont autorisés à condition de conserver un caractère d'aménagement léger. Le schéma de cohérence territoriale (ou une directive territoriale d'aménagement) peut identifier un espace remarquable ayant été façonné par une activité (l'agriculture en particulier) ou entretenu par un gestionnaire d'écosystème (la conchyliculture par exemple), où il conviendra que les plans locaux d'urbanisme n'interdisent pas l'exercice de cette activité. "
} 
Les schémas de mise en valeur de la mer (SMVM) constituent des documents d'urbanisme particuliers à la fois dans leur objet comme dans leur régime juridique $\left({ }^{57}\right)$. Prévus à l'origine par l'article 57 de la loi du 7 janvier 1983 (mais non intégrés dans le droit de l'urbanisme tout en étant des documents d'urbanisme) afin de favoriser la " gestion intégrée et durable » du littoral, les SMVM restèrent pratiquement inusités $\left({ }^{58}\right)$. Le législateur chercha néanmoins à remédier à cette situation par l'article 235 de la loi du 23 février 2005 par un rapprochement entre les SMVM et les SCOT. Cependant, contrairement à ce que d'aucuns auraient pu souhaiter, les SMVM n'ont pas été « absorbés » par les SCOT mais se répartissent désormais en deux catégories : les SMVM de l'article 57 de la loi du 7 janvier 1983, élaborés pour le compte de l'État par le préfet (et produisaient les mêmes effets que les directives territoriales d'aménagement) et ceux de l'article L. 122-1 du Code de l'urbanisme élaborés dans le cadre d'un SCOT incluant une ou plusieurs communes littorales par les EPCl compétents $\left({ }^{59}\right)$.

Le schéma d'aménagement de la plage est une autre forme, certes plus limitée, de réflexion globale sur le devenir de la plage. Décrié à son origine $\left({ }^{60}\right)$, principalement en raison de son caractère circonstancié $\left({ }^{61}\right)$, ce schéma prévu aux articles L. 146-6-1 et R. 146-3 et 4 a été institué « Afin de réduire les conséquences sur une plage et les espaces naturels qui lui sont proches de nuisances ou de dégradations sur ces espaces, liées à la présence d'équipements ou de constructions réalisés avant l'entrée en vigueur de la loi n 86-2 du 3 janvier 1986 " ( $\left.{ }^{62}\right)$.

Mais c'est sans doute en raison des possibilités de maintien d'un certain nombre de construction ou installation des plagistes que ce texte fait débat ${ }^{63}$ ). L'article L. 146-6-1 dispose en effet que "Afin de réduire les nuisances ou dégradations mentionnées au premier alinéa et d'améliorer les conditions d'accès au domaine public maritime, il peut, à titre dérogatoire, autoriser le maintien ou la reconstruction d'une partie des équipements ou constructions existants à l'intérieur de la bande des cent mètres définie par le III de l'article L. 146-4, dès lors que ceux-ci sont de nature à permettre de concilier les objectifs de préservation de l'environnement et d'organisation de la fréquentation touristique. " Pour autant ce n'est pas la seule fonction de ce schéma puisque l'article R. 146-6 lui fixe un large contenu : II "Comporte, pour le territoire qu'il délimite, une analyse de l'état initial du site, portant notamment sur les paysages, les milieux naturels, les conditions d'accès au domaine public maritime et les équipements et constructions réalisés avant l'entrée en vigueur de la loi $n^{\circ} 86-2$ du 3 janvier 1986 relative à l'aménagement, la protection et la mise en valeur du littoral "; " Définit les conditions d'aménagement des plages et des espaces naturels qui leur sont proches ainsi que les modalités de desserte et de stationnement des véhicules. II fixe les mesures permettant d'améliorer l'accès au domaine public maritime, de réduire les dégradations constatées et d'atténuer les nuisances 》; "Détermine, dans la bande des 100 mètres mentionnée au III de l'article L. 146-4, les équipements ou constructions dont le maintien ou

\footnotetext{
${ }^{57}$ S. TRAORÉ, « Les SMVM dans leurs rapports avec les autres documents d'urbanisme . - Commentaire de l'arrêt du Conseil d'État du 3 mars 2008, n²78168, Mme Laporte et autres : JurisData $n^{\circ}$ 2008-073184 ", Construction - Urbanisme $n^{\circ} 1$, Janvier 2009, étude 1.

${ }^{58}$ Seuls quatre SMVM furent élaborés : SMVM de l'étang du Thau (décret du 20 avril 1995 portant approbation du schéma de mise en valeur de la mer du bassin de Thau et de sa façade maritime, JO 21 avr. 1995) ; SMVMdu bassin d'Arcachon (décret $n^{\circ} 2004$ 1409 du 23 décembre 2004, portant approbation du schéma de mise en valeur de la mer du bassin d'Arcachon, JO 28 déc. 2004) ; SMVM du golfe du Morbihan approuvé par le préfet le 10 février 2006 selon les nouvelles modalités prévues par la loi du 25 février 2005 relative au développement des territoires ruraux qui a modifié les articles L. 122-1 et suivants du code de l'urbanisme ; SMVM du Trégor Goélo approuvé le 3 décembre 2007 par le préfet des Côtes- d'Armor.

${ }^{59}$ S. TRAORÉ, « Les SMVM dans leurs rapports avec les autres documents d'urbanisme . - Commentaire de l'arrêt du Conseil d'État du 3 mars 2008, n²78168, Mme Laporte et autres : JurisData n²008-073184 », op. cit.

${ }^{60}$ Gilles GODFRIN, « Un décret pour la plage de Pampelonne », Construction - Urbanisme n 4, Avril 2007, comm. 79.

${ }^{61}$ L'article L.146-6-1 résulte d'un amendement déposé par un député du Var afin de permettre de trouver une solution à la situation de la plage de Pampelonne, située sur le territoire de la Commune de Ramatuelle, dont le projet de réhabilitation soutenu par l'Etat avait été annulé par le juge administratif en première instance (TA Nice, 4 juill. 1996, Assoc. Vivre dans la presqu'île de Saint-Tropez, JCP G 1996, II, 22691, concl. N. Caldéraro ; J.-F. Struillou, Pampelonne, La plage de la discorde : Études foncières, déc. 1997, p. 8), ce qui avait été confirmé en appel (CAA Marseille, 20 janv. 2000, Cne Ramatuelle : BJDU 2-2000, p. 87, concl. L. Benoît) puis devant le Conseil d'Etat (CE, 13 nov. 2002, n² 219034, Cne de Ramatuelle, op. cit.)

${ }^{62}$ Article L. 146-6-1, alinéa 1.

63 Le Schéma « Plans Plages » élaboré à partir de 2009 sur l'ensemble du littoral aquitain, mais dans un cadre juridique différent, n'a semble-t-il pas soulevé les mêmes inquiétudes. Sans doute que le caractère limité des activités de restauration qui s'y pratiquent n'y est pas étranger.

JPF - Colloque du 9 avril 2013 : "Sous la plage, le droit "

Centre de Droit et de Politique Comparés CDPC Jean-CIaude Escarras
} 
la reconstruction peuvent être autorisés par dérogation aux articles L. 146-1 à L. 146-6, ainsi que leur implantation. II indique ceux qui doivent être démolis et fixe les conditions de la remise en état du site. Le schéma d'aménagement définit dans un chapitre distinct les prescriptions qui pourront être imposées aux bénéficiaires des autorisations prévues à l'alinéa précédent afin que ces équipements et constructions ne dénaturent pas le caractère du site et ne compromettent pas la préservation des paysages et des milieux naturels. »

Ce document pourtant d'un intérêt indéniable reste cependant totalement inutilisée ; seule la Commune de Ramatuelle semble en avoir engagé l'élaboration et, courant 2013, reste encore en attente du décret d'approbation en Conseil d'Etat. Outre sans doute les difficultés communes à l'élaboration de l'ensemble des documents locaux d'urbanisme prévisionnel ainsi qu'une certaine frilosité des élus à s'engager dans des processus globaux qui apparaissent trop contraignants, l'exigence démesurée d'un décret d'approbation en Conseil d'Etat démontre la volonté d'en faire un document exceptionnel.

\section{B. La plage dans les documents d'urbanisme généraux}

Parmi les documents d'urbanisme généraux susceptibles de contribuer à la protection de la plage, il convient de distinguer les directives territoriales d'aménagement : DTA (C. urb., art. L. 111-1-1 et L. 146-1) ; les schémas de cohérence territoriale : SCOT (C. urb., art. L. 122-1 et s.) ; les plans locaux d'urbanisme : PLU (C. urb., art. L. 123-1 et s.) ; les cartes communales (C. urb., art. L. 124-1 et s.). Tout en se voyant attribuer un rôle spécifique dans la protection du littoral, ces différents documents d'urbanisme sont chargés par l'article L. 146-6 de " préserver les espaces terrestres et marins, sites et paysages remarquables ou caractéristiques du patrimoine naturel et culturel du littoral, et les milieux nécessaires au maintien des équilibres biologiques. " parmi lesquels l'article R. 146-1 vise notamment "Les dunes, les landes côtières, les plages et les lidos, les estrans ". En outre, ce même article R. 146-1 impose aux documents d'urbanisme ainsi visés "Lorsqu'ils identifient des espaces ou milieux relevant du présent article, les documents d'urbanisme précisent, le cas échéant, la nature des activités et catégories d'équipements nécessaires à leur gestion ou à leur mise en valeur notamment économique ". Il y a là, en matière de plage, des objectifs communs à l'ensemble des documents même si chacun dispose de ses moyens propres d'y parvenir.

Quatre DTA précisent ainsi les modalités d'application de la loi « Littoral », adaptées aux particularités géographiques locales : Alpes Maritimes (approuvée en 2003), Estuaire de la Loire (2006), Estuaire de la Seine (2006) et Bouches du Rhône (2007). Ces DTA s'imposent non seulement aux documents de planification mais également aux opérations. L'article L. 111-1-1 du code de l'urbanisme $\left({ }^{64}\right)$ prévoyait que ces DTA « peuvent également préciser pour les territoires concernés les modalités d'application des dispositions particulières aux zones de montagne et au littoral figurant aux chapitres V et VI du titre IV du présent livre, adaptées aux particularités géographiques locales». II faut noter que seules ces « anciennes » DTA avaient la possibilité de préciser les modalités d’application de la loi « Littoral ». Les « nouvelles » Directives Territoriales d’Aménagement et de Développement Durables (DTADD), issues de la loi n²010-788 du 12 juillet 2010 portant engagement national pour l'environnement, dite " Grenelle 2 », ne disposent plus directement de cette possibilité et non plus la même portée prescriptive, ce qui représente sur ce point un très net retour en arrière. En effet, dans le cas des plages, ces DTA, du fait de leur périmètre essentiellement départemental atteignaient la taille critique qui manque encore parfois aux SCOT littoraux et qui permet sur une bande littorale importante de proposer une vision globale de l'équilibre entre protection et aménagement. Cette compétence reconnue par l'ancien article L. 111-1-1 avait néanmoins ses limites. II ne s'agissait pas de permettre aux DTA de remettre en cause l'application de la loi Littoral ni même d'y déroger mais d'appliquer globalement sur le territoire couvert les principes de la loi.

Depuis la loi n²010-788 du 12 juillet 2010 portant engagement national pour l'environnement qui fait perdre aux DTA ce rôle important, les SCOT littoraux constituent désormais l'un des document les plus prometteurs en matière de

\footnotetext{
${ }^{64}$ Dans sa rédaction antérieure à la loi n²010-788 du 12 juillet 2010 portant engagement national pour l'environnement.
} 
protection du littoral et, spécifiquement, de la plage. En effet, à ce jour la quasi-totalité des communes littorales sont désormais couvertes par un SCOT ${ }^{65}$ )

En outre, en vertu de l'article L.122-1-11 du code de l'urbanisme $\left({ }^{66}\right)$, les SCoT littoraux peuvent comprendre un chapitre individualisé valant SMVM. La finalité du SCOT n'est plus alors seulement de planifier l'aménagement terrestre du territoire concerné mais également de gérer l'interface terre-mer dont la plage est l'un des éléments essentiels. Le contenu du volet littoral d'un SCOT comporte donc alors non seulement des orientations générales pour la régulation des activités présentes sur l'interface terre-mer, mais aussi des sujétions particulières lorsqu'elles sont nécessaires à la protection du milieu marin et littoral, et particulièrement au maintien des équilibres biologiques $\left({ }^{67}\right)$.

Le décret du 5 décembre 1986 relatif au contenu et à l'élaboration des SMVM, dans son article 3, alinéa 3, charge le rapport de présentation d'un SMVM de mentionner les projets d'équipement et d'aménagement liés à la mer tels que les créations et extensions de ports et les installations industrielles et de loisirs, en précisant leur nature, leurs caractéristiques et leur localisation ainsi que les normes et prescriptions spéciales s'y rapportant. Lorsqu'il en va ainsi, c'est donc le contenu du Document d'Orientations et de d'Objectifs (DOO) qui en est principalement affecté, les différents textes régissant un tel schéma de mise en valeur prévoyant l'obligation de mentionner ces diverses orientations $\left({ }^{68}\right)$.

Plusieurs régles spécifiques ou non au PLU sont susceptibles également d'avoir une influence sur la protection des plages par celui-ci. Tout d'abord, en vertu de l'article L. 146-4-III du code de l'urbanisme : « Le plan local d'urbanisme peut porter la largeur de la bande littorale visée au premier alinéa du présent paragraphe à plus de cent mètres, lorsque des motifs liés à la sensibilité des milieux ou à l'érosion des côtes le justifient. »

\footnotetext{
${ }^{65}$ CETE Méditerranée, SCOT littoraux et Grenelle de la mer - La prise en compte des risques littoraux, Février 2012.

${ }^{66}$ Article L. 122-1-11 du code de l'urbanisme : « Lorsqu'ils comprennent une ou des communes littorales, les schémas de cohérence territoriale peuvent comporter un chapitre individualisé valant schéma de mise en valeur de la mer (SMVM) tel que défini par l'article 57 de la loi $n^{\circ}$ 83-8 du 7 janvier 1983 relative à la répartition de compétences entre les communes, les départements, les régions et l'État, à condition que celui-ci ait été approuvé selon les modalités définies au présent chapitre. "

Art. R. 122-3 : [...] Lorsque le schéma de cohérence territoriale comporte un chapitre individualisé valant Schéma de mise en valeur de la mer, [...] II mentionne les orientations relatives aux cultures marines et aux activités de loisirs. II précise dans une perspective de gestion intégrée de la zone côtière, les vocations des différents secteurs de l'espace maritime, les conditions de la compatibilité entre les différents usages de ces derniers, et les conséquences qui en résultent pour l'utilisation des diverses parties du littoral qui sont liées à cet espace. II précise les mesures de protection du milieu marin. Il définit les orientations et principes de localisation des équipements industriels et portuaires, s'il en est prévu.

Il comprend également les dispositions prévues par le décret $n^{\circ} 86-1252$ du 5 décembre 1986 relatif au contenu et à l'élaboration du Schéma de mise en valeur de la mer lorsqu'elles ne sont pas prévues par ailleurs dans le document. [...].

Décret $n^{\circ}$ 86-1252, art. 3 : "Il définit et justifie les orientations retenues en matière de développement, de protection et d'équipement à l'intérieur du périmètre. A cet effet, il détermine la vocation générale des différentes zones, et notamment de celles qui sont affectées au développement industriel et portuaire, aux cultures marines et aux activités de loisirs. II précise les vocations des différents secteurs de l'espace maritime et les conséquences qui en résultent pour l'utilisation des diverses parties de littoral qui lui sont liées. Il définit les conditions de la compatibilité entre les différents usages de l'espace maritime et littoral.

${ }^{67}$ Article R. 122-3, op. cit. ; DDE de l'Hérault, Réaliser un volet littoral et maritime d'un SCOT-Questions Réponses pour avancer dans le projet - Contribution des acteurs du Languedoc-Roussillon à la construction d'une doctrine nationale, novembre 2009.

${ }^{68}$ Outre l'art. R. 122-3 précité, l'article 3 du décret $n^{\circ}$ 86-1252 prévoit qu'il « définit et justifie les orientations retenues en matière de développement, de protection et d'équipement à l'intérieur du périmètre. A cet effet, il détermine la vocation générale des différentes zones, et notamment de celles qui sont affectées au développement industriel et portuaire, aux cultures marines et aux activités de loisirs. II précise les vocations des différents secteurs de l'espace maritime et les conséquences qui en résultent pour l'utilisation des diverses parties de littoral qui lui sont liées. II définit les conditions de la compatibilité entre les différents usages de l'espace maritime et littoral. II mentionne les projets d'équipement et d'aménagement liés à la mer tels que les créations et extensions de ports et les installations industrielles et de loisirs, en précisant leur nature, leurs caractéristiques et leur localisation ainsi que les normes et prescriptions spéciales s'y rapportant. II précise les mesures de protection du milieu marin. II peut prescrire des sujétions particulières portant sur des espaces maritime, fluvial et terrestre attenant, si elles sont nécessaires à la préservation du milieu marin et littoral et particulièrement au maintien des équilibres biologiques. "
} 
Cette disposition a un double intérêt. D'une part, elle permet de substituer à un critère fixe $(100 \mathrm{~m})$ dont les résultats sont instables et évolutifs un critère nettement plus opérationnel comme, par exemple, une projection centennale du trait de côte. Un tel critère permet en outre de prendre en compte, au titre des risques naturels prévisibles visés à l'article L. 121-1 $3^{\circ}$ du code de l'urbanisme, le risque de submersion marine pour lequel le PLU reste un document irremplaçable. D'autre part elle permet alors de justifier pleinement la matérialisation de cette bande issue de I'application de ce critère. Comme le signale L. Prieur, dans la notice du Gridauh sur les PLU littoraux $\left({ }^{69}\right)$ la question de la représentation des limites des espaces est très régulièrement posée. Le plus souvent ces interrogations concernent la représentation de la bande de cent mètres mais la problématique est la même pour l'ensemble des espaces spécifiques issus de la loi Littoral. Pourtant, comme le remarque L. Prieur, « II n'existe aucune obligation de représenter les espaces visés par les dispositions particulières au littoral dans les documents graphiques ou dans les annexes du plan local d'urbanisme. Les articles R. 123-11 à R. 123-13 du code de l'urbanisme qui énumèrent les différentes mentions pouvant figurer dans le PLU ne visent pas les espaces issus de la loi Littoral. » $\left({ }^{70}\right)$.

Par ailleurs, conformément à l'article L. 146-5, il appartient spécifiquement au PLU de délimiter les secteurs dans lesquels, en dehors des espaces urbanisés, l'aménagement et l'ouverture des terrains de campings ou de stationnement de caravanes est possible. Quant on sait les effets que de tels équipements, à proximité des plages, sont susceptibles d'avoir non seulement sur la plage elle-même mais aussi sur les espaces dunaires qui lui sont proches, cette compétence est d'importance.

Enfin, l'article R. 123-8 qui précise que "les zones naturelles et forestières sont dites "zones N". Peuvent être classés en zone naturelle et forestière les secteurs de la commune, équipés ou non, à protéger en raison soit de la qualité des sites, des milieux naturels, des paysages et de leur intérêt, notamment du point de vue esthétique, historique ou écologique, soit de l'existence d'une exploitation forestière, soit de leur caractère d'espaces naturels " ainsi que l'article R. 123-11 qui impose aux documents graphiques de faire apparaître dans son $b$ "les secteurs où les nécessités du fonctionnement des services publics, de l'hygiène, de la protection contre les nuisances et de la préservation des ressources naturelles ou l'existence de risques naturels, tels qu'inondations, incendies de forêt, érosion, affaissements, éboulements, avalanches, ou de risques technologiques justifient que soient interdites ou soumises à des conditions spéciales les constructions et installations de toute nature, permanentes ou non, les plantations, dépôts, affouillements, forages et exhaussements des sols » et dans son $c$ " les espaces et secteurs contribuant aux continuités écologiques et à la trame verte et bleue » donnent au PLU dans le cadre de la délimitation de son zonage les moyens de renforcer la protection non seulement des plages mais surtout de leurs abords immédiats.

En conclusion, il convient de noter que la protection de la plage par le droit de l'urbanisme apparaît de prime abord non seulement complète mais très largement suffisante et même parfois redondante. Néanmoins, les nombreux exemples évoqués par le CGEDD et I'IGA dans leur rapport de $2009\left({ }^{71}\right)$ comme la poursuite d'un contentieux important, souvent favorable aux protecteurs de l'environnement, montrent que cette protection est très largement tributaire de la volonté de ceux qui ont pour mission de la mettre en œuvre. Au nom, notamment, d'intérêts

\footnotetext{
${ }^{69}$ Voir sur la question du caractère facultatif de la matérialisation de cette bande littorale dans les PLU, L. Prieur, « L'écriture des plans locaux d'urbanisme littoraux, Fiche 3 : La traduction de la loi littoral dans les PLU littoraux ", GRIDAUH, http://www.gridauh.fr/comptes-rendus-de-travaux/ecriture-des-plu/, consulté le 10 septembre 2013. Ainsi que CGEDD, " Audit thématique sur l'application de la loi Littoral par les services de l’Etat », La Documentation française, Décembre 2012, p.62. Les auteurs du rapport notent ainsi que, pour le département d'Ille-et-Villaine, seuls 3 des 23 PLU littoraux prévoient une délimitation de la bande littorale

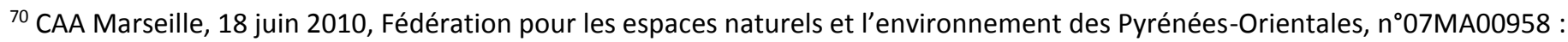
"Considérant que l'absence de matérialisation de la bande littorale de 100 mètres mentionnée par ce texte sur le document graphique du plan d'occupation des sols ne suffit à révéler la méconnaissance par le plan d'occupation des sols de la réglementation relative à l'urbanisation dans ces parties du littoral. "

${ }^{71}$ CGEDD et IGA, op. cit.

JPF - Colloque du 9 avril 2013 : "Sous la plage, le droit "

Centre de Droit et de Politique Comparés CDPC Jean-CIaude Escarras
} 
économiques il est parfois tentant de prendre quelques libertés avec cette exigence, d'autant plus lorsqu'il s'agit de demandes ponctuelles qui ne constituent chacune qu'une atteinte limitée à cette protection mais qui, cumulées, donnent le spectacle cauchemardesque que l'on peut constater sur certaines plages du littoral français. 